\title{
Measuring the impact of ICNIRP vs. stricter-than-ICNIRP exposure limits on QoS and EMF from cellular networks
}

\author{
Jaime Galán-Jiménez ${ }^{\text {a,b,*, Luca Chiaraviglio }}{ }^{\text {c,d }}$ \\ a University of Extremadura, Cáceres, Spain \\ ${ }^{\mathrm{b}}$ Instituto de Investigación en Tecnologías Informáticas Aplicadas de Extremadura, Cáceres, Spain \\ ${ }^{\mathrm{c}}$ EE Department, University of Rome Tor Vergata, Via del Politecnico, 00133, Rome, Italy \\ ${ }^{\mathrm{d}}$ Consorzio Nazionale Interuniversitario per le Telecomunicazioni, Rome, Italy
}

\section{A R T I C L E I N F O}

\section{Keywords:}

Cellular network planning

EMF limits

Impact on QoS

User equipment measurements

ICNIRP

\begin{abstract}
A B S T R A C T
The installation of new equipment (Base Stations, BSs) during the planning phase of a cellular network (including 5G BSs) is governed by exposure limits in terms of allowable ElectroMagnetic Field (EMF) levels. The exposure limits can be either defined by (i) international bodies (e.g., ICNIRP) or (ii) national regulations imposing limits stricter than (i). In this work, we compare the impact of ICNIRP vs. stricter-than-ICNIRP exposure regulations on the Quality of Service (QoS) and EMF. To this aim, we perform a large-scale measurement campaign in one scenario in Spain subject to ICNIRP regulations and another one in Italy subject to EMF limits stricter than ICNIRP ones. Both the scenarios are characterized by similar exposure conditions, comparable user density, and common $4 \mathrm{G}$ performance targets by the operators. Results, obtained by measuring QoS and EMF at selected locations, reveal that the QoS in the scenario subject to strict EMF limits is heavily worsened compared to the one in which ICNIRP-based limits are enforced. Clearly, the scenario with strict EMF limits presents a lower level exposure over the territory compared to the one imposing ICNIRP limits.
\end{abstract}

\section{Introduction}

The evolution of cellular networks, from $2 \mathrm{G}$ to forthcoming $5 \mathrm{G}$, has been driven by the evolving requirements of the applications, whose features are being continuously modified to satisfy customers' demands. The migration from an already-deployed network to a new one must face several challenges (i) planning when and how to introduce novel hardware equipment; (ii) handling the deployment of network services that are currently working towards the new technology; (iii) adapting the network operator policies to the novel technology.

In this paper we study the first challenge, i.e., the installation of new equipment (Base Stations, BS) during the planning phase in the migration from $4 \mathrm{G}$ to $5 \mathrm{G}$ networks. The installation of new BSs must be performed according to several regulations in terms of the ElectroMagnetic Field (EMF) levels, while at the same time ensuring a (possibly) adequate Quality of Service (QoS) to the user. The International Commission on Non-Ionizing Radiation Protection (ICNIRP) imposes a set of limits on the EMF generated by the BSs that are adopted in many countries of the world [1], including e.g., Spain. On the other hand, other countries, e.g., Italy impose EMF limits stricter than ICNIRP [25]. In Italy, for example, a maximum EMF limit of $6[\mathrm{~V} / \mathrm{m}]$ is imposed for the radiation from BSs in residential zones. Moreover, a minimum distance of 100 meters between a BS and a sensitive place is enforced in many cities of the country, such as Rome [3]. On the contrary, no national or local regulations in terms of minimum distance to sensitive places are adopted (e.g., a BS can be installed on top of a hospital or educational center) in Spain. Moreover, a debate on the risks derived by the exposure of people living in vicinity to BSs is gaining momentum in these days [6].

Planning a cellular network under restrictive EMF constraints is not optimal from the point of view of the QoS perceived by the user, as clearly shown by previous works [7-9]. However, different questions emerge are still not answered by previous works, including: (i) Which is the impact of applying different EMF regulations on the QoS perceived by the user? (ii) Which is the impact in terms of EMF generated by the User Equipment (UE) in such cases? The goal of this paper is to shed light on these issues. In particular, a comparison of the cellular network planning carried out in two countries with different EMF restrictions (Spain - ICNIRP, and Italy, Stricter-than-ICNIRP) is performed. To this aim, we perform a thorough analysis from a purely technical perspective to compare the network planning of already-deployed 4G cellular networks in two scenarios which are comparable in terms of terrain

\footnotetext{
* Correspondence to: Department of Computer Systems and Telematics Engineering, University of Extremadura, Cáceres, Spain. E-mail addresses: jaime@unex.es (J. Galán-Jiménez), luca.chiaraviglio@uniroma2.it (L. Chiaraviglio).
} 


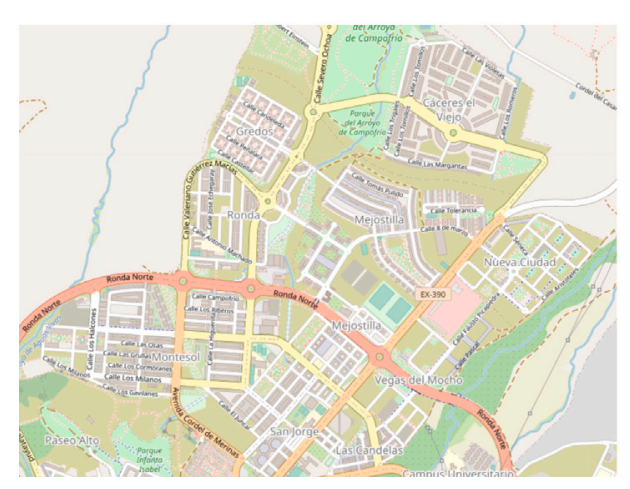

(a) Cáceres $(\mathrm{CC})$

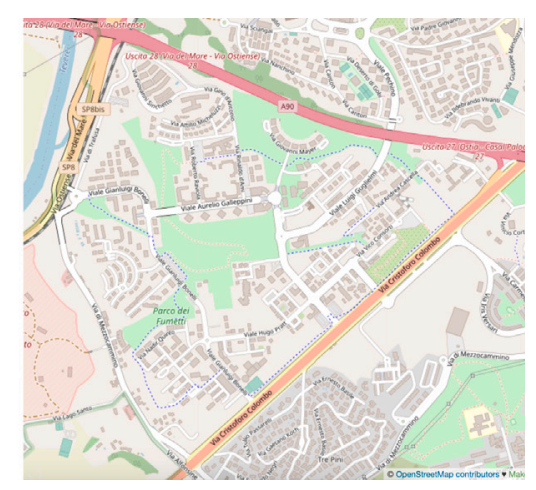

(b) Torrino Mezzocamino (TMC)

Fig. 1. Targeted scenarios.

dimensions and population density, but very different in terms EMF regulations.

The rest of the paper is organized as follows. Section 2 reviews the background, focusing on the ICNIRP vs. Non-ICNIRP regulations. Section 3 reports a quantitative analysis of QoS metrics experienced at the user side, whilst a qualitative one of the EMF generated by both the BS and the UE is reported in Section 4. A set of multivariate analyses are carried out and described in Section 5 to show the effectiveness of the measurement campaigns performed. Finally, Section 6 concludes our work and introduces future research lines.

\section{Background: Regulations on EMF emissions}

It is well known that a high exposure to high levels of EMF is dangerous for human health. The debate on the health implications of EMF levels close to the ICNIRP limits is still ongoing within the research community [10-13]. Moreover, the implications of future 5G BSs deployment on the potential risks of the EMF emissions on human health have recently become a concern for society [14]. As conclusion of this work, the authors state that there are no scientific evidences that support the controversy generated by the fact of installing $5 \mathrm{G}$ BSs in dense populated areas.

Strict regulations are imposed to limit the amount of time a person should be exposed to particular EMF levels generated by radiofrequency and electrical sources, such as, e.g., smartphones, tablets or laptops. However, there is not an unique worldwide regulation, and it varies across countries. In this work, we focus on two European countries: Spain and Italy. These territories, although they are in a relative close proximity, different EMF regulations exist for them.

\subsection{ICNIRP vs Non-ICNIRP EMF limits}

Most of the European countries, including Spain, follow the limits defined by the ICNIRP [15]. ${ }^{1}$ A different (and most restrictive regulation) is applied in Italy, where the type of territory is also taken into account for setting the EMF level limits. As an example, a residential area in which people typically stand for a long time (home, work) is considered different from a transitory public one (park, hospital, or similar), where people do not spend a high amount of time. Moreover, sensitive places such as hospitals, public parks or schools are prevented from the installation of nearby BSs, having a minimum limit of at least 100 meters between the BS site and the target place.

Table 1 reports a list with a set of countries and the regulation that is applied on them. In particular, we focus on Spain and Italy, which are

1 For frequencies higher than $2 \mathrm{GHz}$, power density should be used instead of incident Electric strength.
Table 1

List of a set of countries with the adopted regulations and further constraints.

\begin{tabular}{lll}
\hline Country & Reference & Regulation and Constraints \\
\hline Canada & {$[16]$} & Non-ICNIRP, Lower Max EMF \\
China & {$[17]$} & Non-ICNIRP, Lower Max EMF \\
Greece & {$[16]$} & Non-ICNIRP, Lower Max EMF, Min Distance \\
Italy & {$[3,4,16,18]$} & Non-ICNIRP, Lower Max EMF, Min Distance \\
Spain & {$[15]$} & ICNIRP limits \\
Switzerland & {$[5]$} & Non-ICNIRP, Lower Max EMF, Min Distance \\
\hline
\end{tabular}

the main target of our work. It can be seen that the ICNIRP regulation is adopted in Spain and a more strict one is imposed in Italy. Nevertheless, other countries are also listed to show the different constraints that are applied, such as (i) lower maximum EMF levels compared to the ones adopted by ICNIRP, and (ii) minimum distance is required between a BS and a sensitive public place, such as hospitals, parks, etc.

In the following, a comparison between the two considered sets of limits, i.e., ICNIRP and Non-ICNIRP-based countries, is performed as a function of the frequency at which the EMF is generated. By inspecting Table 2, Italy regulation is in general stricter than the ICNIRP one. Moreover, for residential areas, it is even more restrictive, having a maximum limit of $6[\mathrm{~V} / \mathrm{m}]$ for all the frequencies. It is worth to say that if we focus on the specific city in which our analysis is performed (i.e., Rome), the local regulation [3] imposes additional rules such as the minimum distance of 100 [m] between a potential BS site and a sensitive place (as already introduced in Section 2), which is evaluated on a case-by-case basis. Indeed, other type of limitations may refer to the type of area in which the BS is installed, such as, e.g., the ancient part of a city or an area protected to animals. Moving our attention to the Spanish scenario, it can be seen in Table 2 that the regulation adopted is the one defined by the ICNIRP, with no specific limitations as in the case of Italy. As a summary, the Italian limits are much more stringent compared to the ICNIRP-based ones (e.g., Spain).

\section{Quantitative analysis of QoS metrics at UE}

In this section, a quantitative analysis of the results obtained during a measurement campaign in each scenario is described. The first analysis that is proposed compares the impact of the cellular planning on ICNIRP and Non-ICNIRP-based countries, with regard to different QoS metrics. Such considered metrics are the Reference Signal Received Power (RSRP), the number of different BSs and cell IDs ${ }^{2}$ the UE is receiving signal from, and the distance from the serving BS. The structure of the section is as follows. At first, the main features of the two target scenarios are described in Section 3.1. Next, the methodology followed

\footnotetext{
2 The cell ID is a unique identifier of a BS sector [19].
} 
Table 2

EMF regulations for the cellular planning across the different scenarios.

\begin{tabular}{lll}
\hline Type of area & ICNIRP (e.g., Spain) [15] & Non-ICNIRP (e.g., Italy) \\
\hline \multirow{3}{*}{ Public (transitory) } & $28[\mathrm{~V} / \mathrm{m}], f \in(10,400][\mathrm{MHz}]$ & $60[\mathrm{~V} / \mathrm{m}], f \in(0.1,3][\mathrm{MHz}]$ \\
& $1.375 \cdot f^{1 / 2}[\mathrm{~V} / \mathrm{m}], f \in(400,2000][\mathrm{MHz}]$ & $20[\mathrm{~V} / \mathrm{m}], f \in(3,3000][\mathrm{MHz}]$ \\
& $61[\mathrm{~V} / \mathrm{m}], f \in(2,300][\mathrm{GHz}]$ & $40[\mathrm{~V} / \mathrm{m}], f \in(3,300][\mathrm{GHz}]$ \\
\hline & $28[\mathrm{~V} / \mathrm{m}], f \in(10,400][\mathrm{MHz}]$ & \\
Residential (steady) & $1.375 \cdot f^{1 / 2}[\mathrm{~V} / \mathrm{m}], f \in(400,2000][\mathrm{MHz}]$ & $6[\mathrm{~V} / \mathrm{m}] \forall f$ \\
& $61[\mathrm{~V} / \mathrm{m}], f \in(2,300][\mathrm{GHz}]$ & \\
\hline
\end{tabular}

Table 3

Features of the Cáceres (CC) 3rd North District scenario [20].

\begin{tabular}{llll}
\hline Neighborhood & Inhabitants & Area & Perimeter \\
\hline Montesol & 4,126 & $0.77 \mathrm{~km}^{2}$ & $4.5 \mathrm{~km}$ \\
Mejostilla & 5,520 & $0.78 \mathrm{~km}^{2}$ & $4.5 \mathrm{~km}$ \\
Ronda & 1,386 & $0.30 \mathrm{~km}^{2}$ & $2.6 \mathrm{~km}$ \\
Gredos & 1,539 & $0.19 \mathrm{~km}^{2}$ & $1.7 \mathrm{~km}$ \\
\hline
\end{tabular}

to perform the measurements is explained in Section 3.2. Finally, a discussion of the results extracted from the measurements is included in Section 3.3.

\subsection{Scenarios}

Two scenarios have been considered for the quantitative comparison. The first scenario is represented in Fig. 1(a) and refers to the 3rd North District of Cáceres (Spain), composing 4 neighborhoods of relatively recent creation, namely Montesol, Mejostilla, Ronda, and Gredos. An area of $4.08 \mathrm{~km}^{2}$ is covered, with a population of 12,571 inhabitants in 2019 [20]. It is a residential district located in the surroundings of Cáceres and it is composed of single family houses, small flats up to 3 floors at most, and large public green areas. Table 3 shows the information of the targeted district, which is retrieved from [20]. In this scenario, three different operators providing LTE connectivity are evaluated, namely (i) Movistar; (ii) Vodafone; and (iii) Orange.

The second scenario, which is represented in Fig. 1(b), maps to the Torrino Mezzocammino (TMC) district in Rome (Italy), which is subject to the Italian-wide general (Non-ICNIRP) limits with the local restrictions already reported in Table 2. TMC is also a newly-created district located in the surroundings of Rome, with $5.12 \mathrm{~km}^{2}$ [21] and 14,024 inhabitants at the end of $2018^{3}$ [22]. As in Cáceres, small flats and houses compose the district, where there are also large green parks to do sport. Concerning the set of operators, three operators are again evaluated: (i) TIM; (ii) Vodafone, and (iii) Wind Tre [23].

\subsection{Methodology}

We start the description of the methodology focusing on the Cáceres (CC) scenario. A measurement campaign was carried out throughout the targeted neighborhood during more than one month, starting on May 4th, 2019, and ending on June 10th, 2019. In order to be fair, the same time period has been selected for measuring day by day, i.e. from 6:30 p.m. to 8:30 p.m. In this way, all the measurements can be combined in the same map or figure under the same conditions. For measurements records, three different UE were used: 2 x Motorola Moto G 4 Plus, and one Xiaomi Mi A2. For the setting of the campaign at TMC, we refer the reader to [23], in whose work the same methodology was adopted.

CellMapper [19] and MapMarker [24] apps have been used for the measurement campaigns. We define an interval of one second between two consecutive measurements. Each measurement $m$ at time instant

3 TMC district refers to id 431 in the toponymy subdivision of the city of Rome.
Table 4

Recorded information for each operator in both scenarios.

\begin{tabular}{lll}
\hline Feature & CC (ICNIRP) & TMC (Non-ICNIRP) \\
\hline \multirow{3}{*}{ Operator } & Movistar & TIM \\
& Vodafone & Vodafone \\
& Orange & Wind Tre \\
\hline Period & May 4th, 2019- & May 1st, 2018- \\
& June 10th, 2019 & May 31st, 2018 \\
\hline \multirow{3}{*}{ Number of records } & 10,837 (Movistar) & 8,676 (TIM) \\
& 12,221 (Vodafone) & 13,445 (Vodafone) \\
& 10,513 (Orange) & 10,292 (Wind Tre) \\
\hline \multirow{3}{*}{ UE model } & Xiaomi Mi A2 & Samsung S6 Edge \\
& Motorola Moto G & OnePlus3 \\
& 4 Plus (x2) & Huawei Honor 7 \\
\multirow{2}{*}{ Apps versions } & CellMapper app. v.5.2.7 & CellMapper app. v.5.1.7 \\
& MapMarker app. v.2.17.2 & MapMarker app. v.2.14.1 \\
\hline
\end{tabular}

$t$ is defined by a tuple of type $m(t)=\{p(t), r(t), s(t), c(t), b(t)\}$, where $p(t)$ is the GPS location of the UE; $r(t)$ is the received RSRP value; $s(t)$ is the type of cellular service provided; $c(t)$ represents the cell ID the UE is connected to; and $b(t)$ is the BS ID of the serving BS. This value is obtained by exploiting [25]. Since a huge amount of measurements were obtained, areas of $30 \times 30\left[\mathrm{~m}^{2}\right]$ were defined to compute average values. The reason for performing this is threefold: (i) as the density of the measurements maybe not uniform, the sampling operation allows to work with an uniform grid of areas; (ii) we are able to extract information about the metrics over the territory, e.g., by computing the number of distinct cell IDs and BS IDs that can be measured in an area; (iii) we treat in the same way all the measurements in the same area taken at different time instants. With this aggregation, the computation of the number of distinct cell IDs and BS IDs can better highlight the correctness of the cellular network planning deployed. The size of the areas has been selected with the aim of aggregating a statistically significant number of measurements, as well as being representative for outdoor scenarios.

For the average computation of the RSRP (whose unit is dBs) at each area, the measured value (i.e., RSRP) is firstly translated into linear, then, the average (in linear) is computed, and finally a translation into $\mathrm{dB}$ is again carried out. A total of 33,571 and 32,413 measurements with QoS metrics were taken in CC and TMC, respectively. Pandas Python library [26] was used to parse the raw data outputted by CellMapper and to aggregate them into the defined areas. Table 4 summarizes the information of the measurement campaigns taken in CC and TMC with respect to the analyzed operators, the time period, the number of measurements recorded, the model of UEs used and the apps versions.

\subsection{Measurements characterization}

In this section, we provide an analysis of different QoS metrics based on the measurements obtained during the two campaigns. At first, the values of the RSRP received by the UEs are analyzed. Next, an evaluation of the coverage overlap is performed. Finally, the distance to the serving BS is inspected to determine if there is a significant impact on the RSRP. 


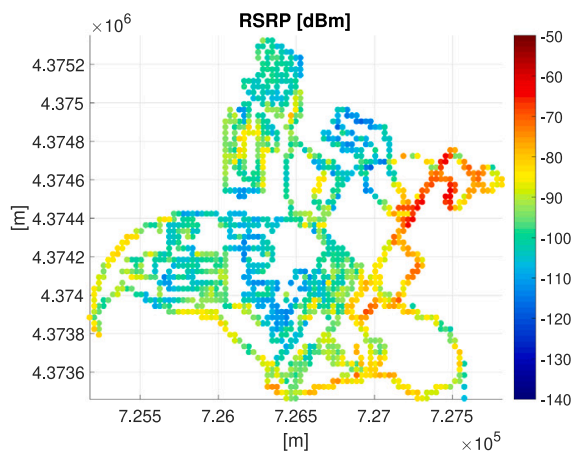

(a) CC - Movistar

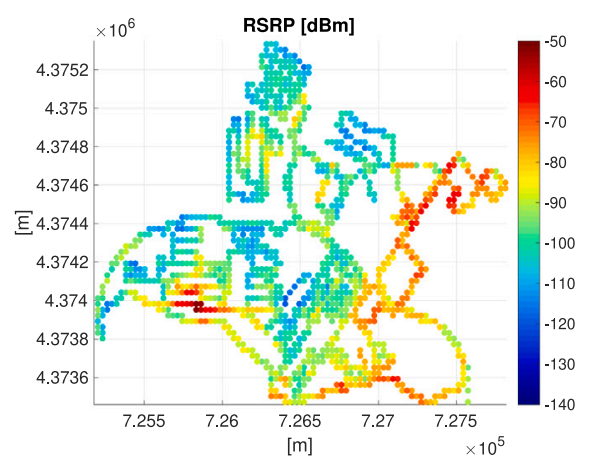

(c) CC - Vodafone

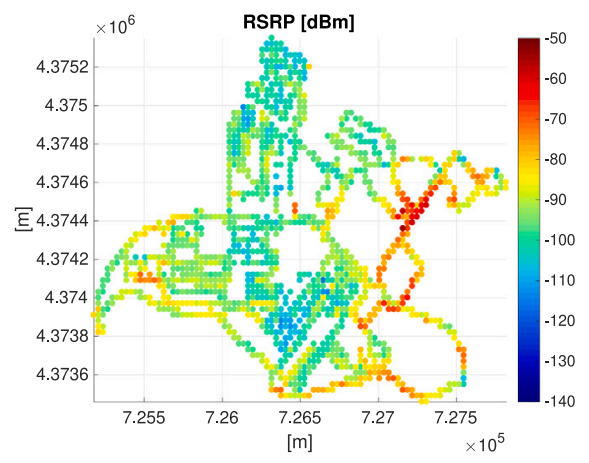

(e) CC - Orange

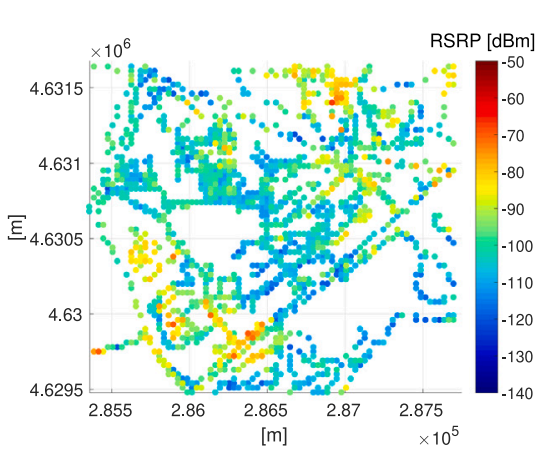

(b) TMC - TIM [9]

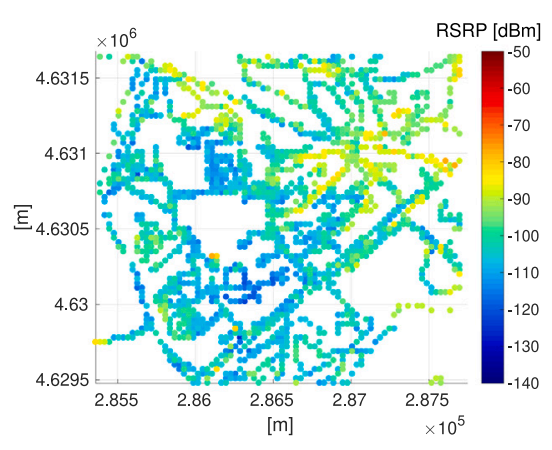

(d) TMC - Vodafone [9]

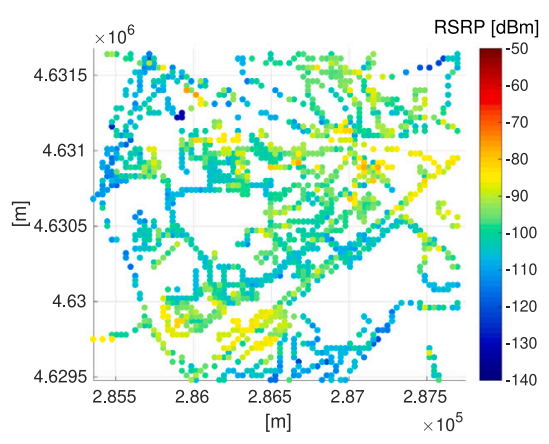

(f) TMC - Wind Tre [9]

Fig. 2. Average RSRP measured over the territory across the different operators and both scenarios (Figures best viewed in colors).

\subsubsection{RSRP results}

Figs. 2(a), 2(c), 2(e) represent the average RSRP per area of $30 \mathrm{x}$ $30\left[\mathrm{~m}^{2}\right]$ for the three operators in the CC scenario, whose resulting color maps the colorbar placed at the right of each subfigure. Looking at the figures, it can be extracted that in general, RSRP values in the CC scenario are higher (better signal strength) compared to the case of TMC [9] (see Figs. 2(b), 2(d), 2(f)), i.e., hotter colors prevail against colder ones. In particular, Vodafone operator provides higher values of RSRP compared to Movistar and Orange. On the contrary, if we move our attention to the TMC district, TIM and Wind Tre seem to provide higher values of RSRP compared to Vodafone. The main reason of this difference between scenarios is the number of BSs located inside both neighborhoods. This information can be gathered by looking at the hottest areas in the figures and therefore by confirming their existence on an on-site check. In CC, apart from one BS site at location (7.273, 4.3744) $[\mathrm{m}]$ providing coverage to the three operators, there is an additional and dedicated BS for Vodafone at $(7.258,4.374)[\mathrm{m}]$, which provides an higher RSRP value in the nearby streets. For BSs location at TMC, we refer the reader to $[9,23]$.
Fig. 3 shows the empirical Cumulative Distribution Function (CDF) of the average RSRP per area for both scenarios. It is clear from the figure that the average RSRP measured in CC are lower compared to TMC for all the operators (blue curves are shifted to the right compared to the red ones). By inspecting the right part of the CDFs, we can note that the best results are obtained by Vodafone and Orange in CC. If the left part of the CDFs are now analyzed, very low values of RSRP are reported for Vodafone and TIM for a significant percentage of areas (e.g., between -107 and $-105[\mathrm{dBm}]$ for the $40 \%$ of the measurements).

As conclusion of the RSRP analysis performed, we can remark that there is a strong difference in the RSRP values obtained in similar neighborhoods located in different countries with different regulations, which can directly affect the perceived QoS by the users in such regions. In our experiments, the ICNIRP scenario (CC) presents, in general, much higher values of RSRP for all the operators compared to the case of the Non-ICNIRP-based scenario (TMC). 


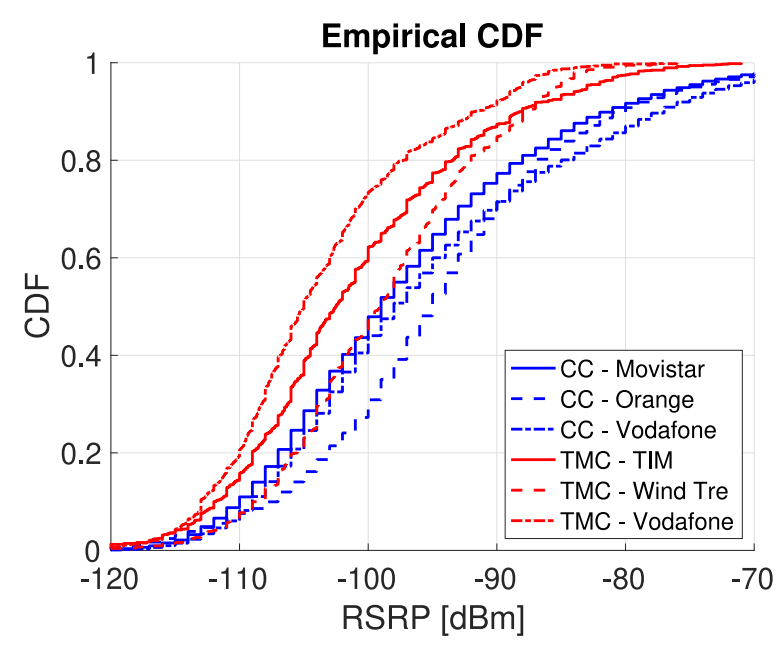

Fig. 3. CDFs of the RSRP values across the different countries and operators.

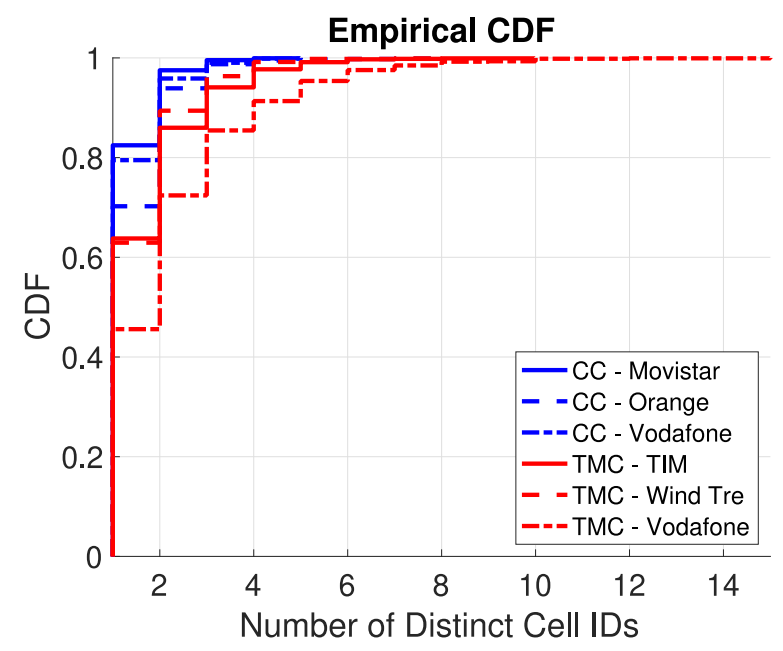

Fig. 4. CDFs of the number of distinct cell IDs across the different countries and operators.

\subsubsection{Coverage overlap results}

The purpose of the next analysis is to inspect if there are also differences in the number of different BSs and cell IDs that are received by a user across the considered scenarios and operators. At first, Fig. 4 shows the empirical CDF of the number of distinct cell IDs that are received within an area of $30 \times 30\left[\mathrm{~m}^{2}\right]$. It can be seen that more than the $70 \%$ of the tested areas in the CC district is covered by only one BS at most for all the operators, while the remaining $30 \%$ of the territory receives signal strength from $2 \mathrm{BS}$ sites at maximum. However, the TMC scenario presents a larger number of cell IDs per area. For instance, if we focus on the Vodafone operator, $55 \%$ of the areas are covered by two or more cell IDs in TMC (30\% with $3+$ cell IDs and, remarkably, $5 \%$ of the areas with $6+$ cell IDs). The latter increase in the number of distinct cell IDs obtained in the TMC scenario can be the result of being placed at the edge of a coverage area, receiving signal strength from different sources. At this point, several questions emerge: (i) is it better from the QoS point of view to be placed in an overlapped area or is it preferred to receive LTE signal from only one source? (ii) a higher value of received RSRP is related to a larger or smaller number of distinct sources? In the following, we aim at answering these and other related questions.

Taking into account that one BS site is composed of several cell IDs, an aggregated analysis is performed to examine the number of

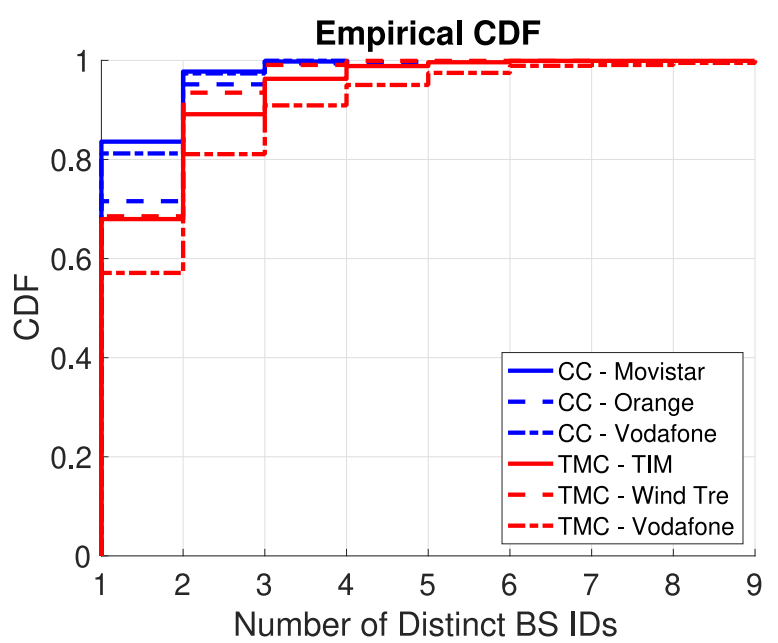

Fig. 5. CDFs of the number of distinct BS IDs across the different countries and operators.

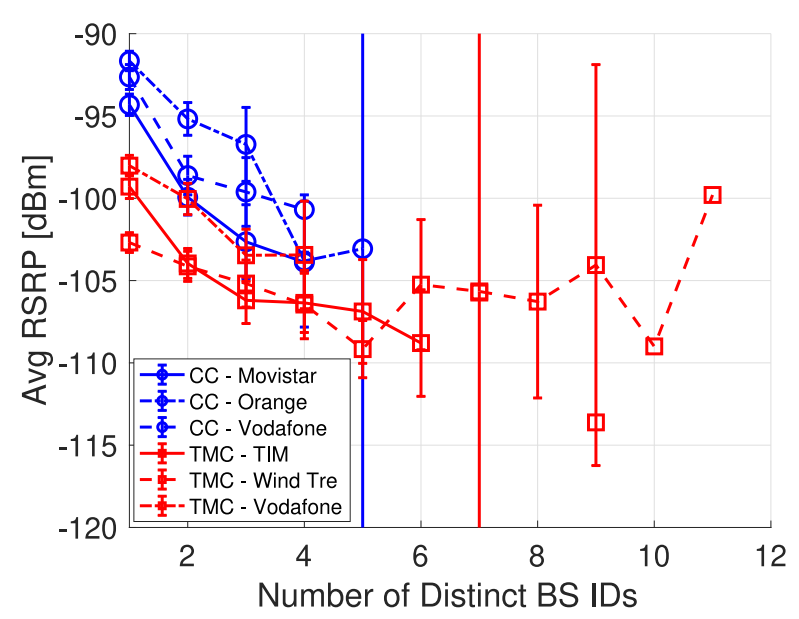

Fig. 6. Average RSRP vs number of distinct BS IDs across the different countries and operators.

distinct BS IDs that are received at one particular area. The outcomes of this analysis are reported in Fig. 5. A very similar trend is depicted by the CDFs when comparing Figs. 4 and 5. Again, the CC district presents lower values for the number of distinct BSs that are perceived in the majority of the territory, i.e., a larger portion of the scenario is covered by a lower number of BS IDs. In particular, Movistar is almost fully-covered by only one BS site ( $82 \%$ of the district) and the highest number of BS IDs received at any place in the CC scenario is 4 . However, this number increases in TMC, especially for Vodafone, with more than $40 \%$ being covered by two different BS sites or more.

A comparison between the average RSRP values and the number of BS IDs obtained in the same area is depicted by Fig. 6, with $95 \%$ confidence intervals. As expected, a decreasing tendency of the average RSRP value is experienced with the increase of the number of distinct BS IDs, i.e., if a higher number of BS sites impacts on an area, the average RSRP is worsening.

Results again confirm that the ICNIRP scenario (CC) presents better RSRP values than the ones obtained in TMC, with an order of about $5[\mathrm{dBm}]$ on average. Moreover, the number of BS IDs per area is in general lower in CC. Moreover, the operator with the largest number of BS IDs in TMC is Vodafone (11), 6 BS sites more than in the worst-case scenario of the CC district (Orange with 5). To sum up, the ICNIRPbased scenario presents higher values of RSRP and less number of cell IDs and BS IDs compared with the Non-ICNIRP-based one. 


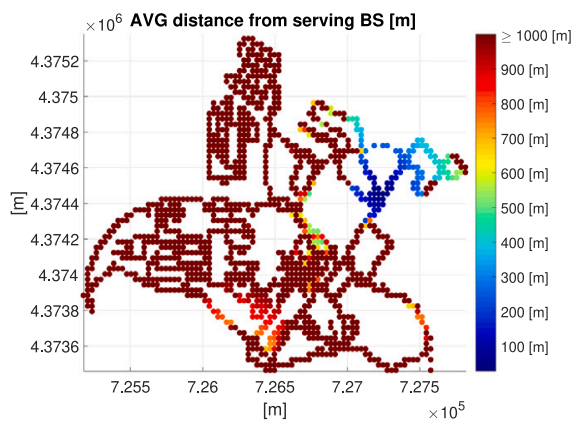

(a) CC - Movistar

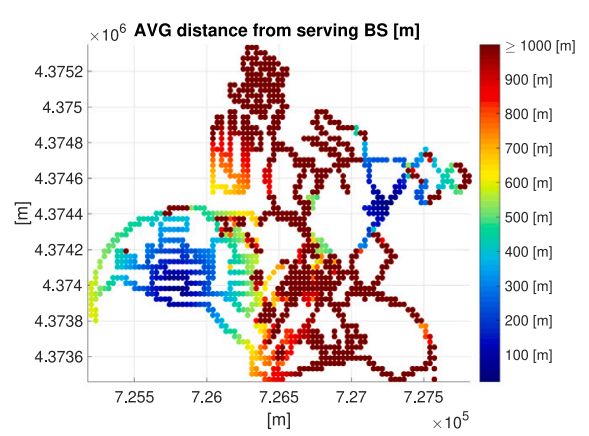

(c) CC - Vodafone

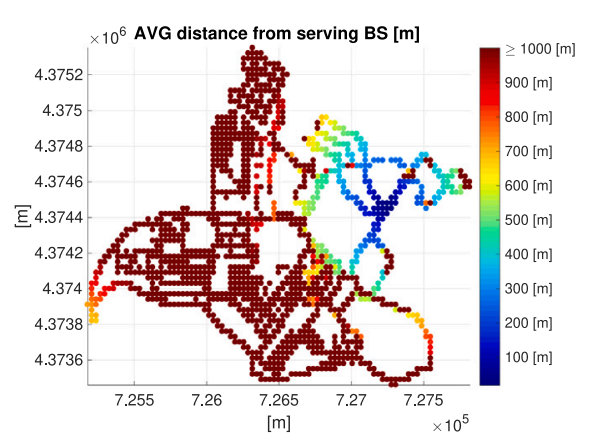

(e) CC - Orange

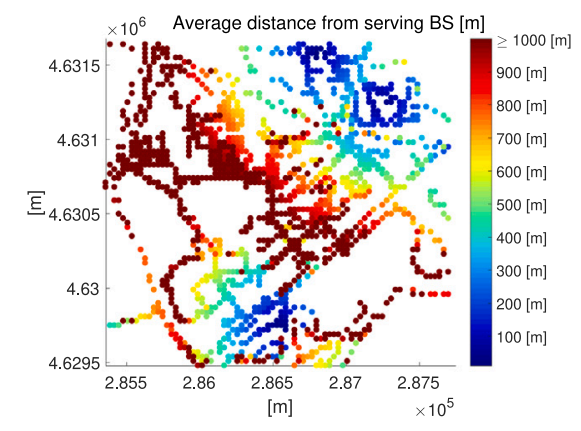

(b) TMC - TIM [9]

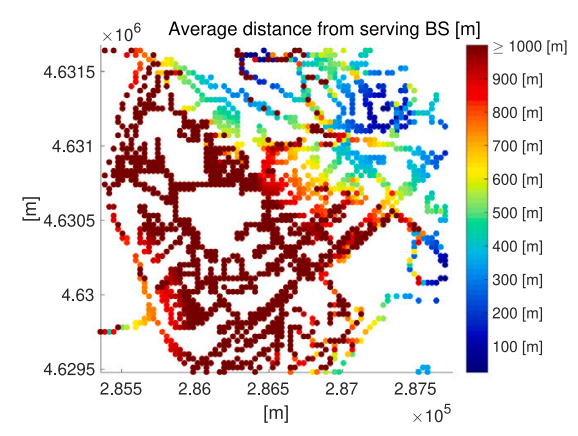

(d) TMC - Vodafone [9]

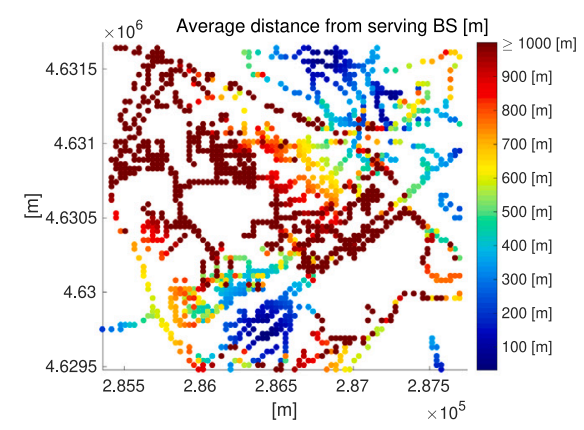

(f) TMC - Wind Tre [9]

Fig. 7. Average distance from Serving BS over the territory across the different operators and both scenarios (Figures best viewed in colors).

\subsubsection{Distance to serving $B S$ results}

Next analysis introduces the distance to the serving BS as a new parameter to be compared with the received RSRP values. In this analysis, our goal is to compare BS-related metrics based on real measurements gathered in the performed campaigns. A first general overview is depicted in Fig. 7, where a colored map is provided for each scenario and each operator. Again, the color code follows the colorbar on the right of each subfigure.

From the set of figures, we can extract that the distance from the serving BS is much more larger in CC than in TMC [9], except for the case of Vodafone in CC, since there is a dedicated BS located in the south west of the district (Fig. 7(c)). The relationship between the average RSRP values and the distance to the serving BS is represented in Fig. 8 for the two scenarios and the six considered operators. As in the case of the evaluation of the RSRP as a function of the number of distinct BS IDs (see Fig. 6), a consistent negative trend in the value of the average RSRP is experienced with the distance from the serving BS for all the operators in both scenarios. If we compare CC with TMC, the former achieves better results in the obtained RSRP when the user is in close proximity $(\leq 500[\mathrm{~m}])$ or far away the serving BS ( $>800[\mathrm{~m}]$ ), especially for Movistar and Orange. In the middle range
(500 $\leq d \leq 800$ ), although it is clear that Orange in CC is the operator that outperforms the rest, the curves for both scenarios are mixed, which means that they behave in a similar way when comparing RSRP and distance. These minor oscillations may emerge since the RSRP is affected by multiple components, including (apart from the distance from the serving BS) effects such as multipath, shadowing/fading, antenna tilting/orientation, etc.

We remark here that, although it would be possible to apply a propagation model to estimate the RSRP and consequently the throughput values, we report evidence that, even by adopting an empirical propagation model, the RSRP alone is not sufficient to derive indications about the performance level. As reported by [27], the SINR (Signal-to-Interference-plus-Noise Ratio), which affects the throughput, is formally expressed as:

$$
S I N R=\frac{R S R P}{(N+I)}
$$

where $N$ is the floor noise, and $I$ is the interference. On the other hand, in close proximity of the BS, the RSRP can be simplified as:

$S I N R \approx \frac{R S R P}{N}$ 
Table 5

Devices and tools to perform the UE EMF evaluation.

\begin{tabular}{llll}
\hline Device & & CC & TMC \\
\hline GPS tracker & & MapMarker app. v.2.17.2 & MapMarker app. v.2.14.1 \\
\hline QoS metrics meter & & CellMapper app. v.5.2.7 & CellMapper app. v.5.1.7 \\
\hline \multirow{2}{*}{ UE } & Model & Motorola Moto G4 Plus & Samsung S6 Edge \\
& Operator & Orange & Vodafone \\
& Operating band & B3 (1800 [MHz]) & B3 $1800[\mathrm{MHz}])$ \\
\hline \multirow{3}{*}{ EMF meter } & Model & PMM 8053 [28] & PCE-EM300 \\
& Resolution & $0.01[\mathrm{~V} / \mathrm{m}]$ & $0.01[\mathrm{~V} / \mathrm{m}]$ \\
& EMF range & $0.3-300[\mathrm{~V} / \mathrm{m}]$ & $0.1-200[\mathrm{~V} / \mathrm{m}]$ \\
& Time granularity & $1[\mathrm{~s}]$ & $1[\mathrm{~s}]$ \\
Test & Frequency range & $100[\mathrm{kHz}]-3[\mathrm{GHz}]$ & $100[\mathrm{kHz}]-3[\mathrm{GHz}]$ \\
& File type & MPEG-4 Video & MPEG-4 Video \\
\hline \multirow{2}{*}{ Selected BS } & File size & $7[\mathrm{MB}]$ & $7[\mathrm{MB}]$ \\
& File transfer & Upload+send a file using Gmail app & Upload+send a file using Gmail app \\
\hline
\end{tabular}

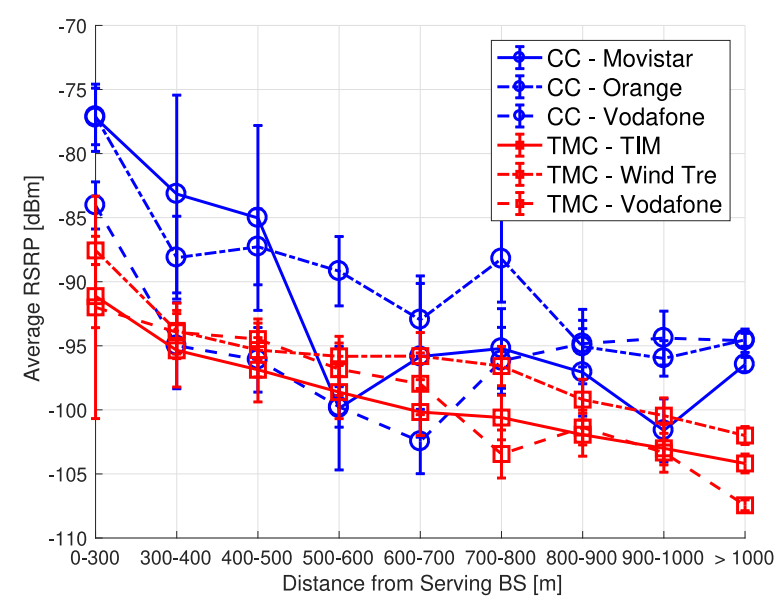

Fig. 8. Average RSRP versus serving distance across the different countries and operators.

In this region, the RSRP is a good indicator of the SINR, and consequently of the performance. However, Eq. (2) does not hold for the whole extent of the sector. In particular, when the UE is located at the cell edge, Eq. (1) holds. In this region, the SINR may be dominated by interference. Therefore, the application of a propagation model to estimate the RSRP (and consequently the SINR), would derive results not consistent with the measured ones.

As a summary, with the reported analysis it is important to observe two main dominating effects: (i) in the proximity to the BS, the RSRP is in general higher in CC compared to TMC, and (ii) for pixels at a great distance from the serving BS (e.g., higher than $1000 \mathrm{~m}$ ), the RSRP is clearly higher in CC compared to TMC. In order to link the experienced QoS with the level of EMF generated by the equipment in use, a qualitative analysis is performed in the next section, comparing the impact of the EMF contributions from both the UE and the serving BS.

\section{Qualitative analysis of EMF generated by UE and perceived QoS}

In the previous section, a quantitative analysis based on the RSRP values obtained by the CellMapper app [19] has been presented as a function of the coverage overlap and the distance to the serving BS. In the following, a qualitative one is carried out to evaluate the impact of the generated EMF when LTE uplink connectivity is used. In particular, we evaluate both the EMF generated by the serving BS and the one produced by the UE. At first, the setting of the tests is described. Then, the breakdown of the results obtained in the measurement campaign are analyzed and statistically validated by means of a set of multivariate analyses.

The target scenario is on-site explored to select a subset of locations in which measurements are taken. Three parameters have been varied to create such subsets: (i) the distance to the serving BS; (ii) the RSRP value; and (iii) the sight condition; i.e., the situation that exists between the UE and the BS (LOS - Line of Sight, SLOS — Semi Line of Sight, and NLOS - No Line of Sight). Fig. 9 shows the terrain view of the considered scenario, where circled numbers represent the ID of the measurement and the pin is placed in the location of the serving BS.

As introduced above, the main goal of the current analysis is to evaluate the impact of the use of the LTE uplink in terms of the EMF that is generated. In both scenarios, we have adopted the same methodology to take the EMF measurements: (i) the sending UE (emitter) is placed at a height of $0.8[\mathrm{~m}]$ with orientation towards the serving BS; (ii) the EMF meter is placed opposite to the UE and in close proximity; (iii) a video of size $7 \mathrm{MB}$ is sent to a specific e-mail account; (iv) the EMF generated by the UE is recorded every second; (v) once the file transfer is finished, the UE is turned off; (vi) the EMF meter is twirled $180^{\circ}$ and pointed towards the serving BS; (vii) the EMF generated by the BS is recorded every second during a time period of $30 \mathrm{~s}$. Table 5 shows a summary of the devices and tools used during the measurements campaign.

Table 6 shows the breakdown of the results obtained in the CC scenario. For each measurement, we report the RSRP value, the distance to the serving BS, the sight condition the measurement was taken under, the average value of the EMF generated by the BS, the average value of the EMF generated by the UE while uploading the file, and the time required to upload the file. From the tables, it can be extracted that a high variability of the RSRP values, the average EMF from the BS and the one generated by the UE is experienced. In the following, several analyses are performed in order to extract certain conclusions when comparing the cellular planning of ICNIRP and Non-ICNIRP-based countries in terms of EMF values.

The first analysis we propose is to compare the impact of the distance to the serving BS and the sight condition on the RSRP values recorded by the UE. Fig. 10 reports the obtained RSRP values as a function of the distance to the serving BS for the two considered scenarios: CC (Fig. 10(a)) and TMC (Fig. 10(b)). The sight condition is represented by different colors (LOS - blue, Semi-LOS - green, and NLOS - red) to better evaluate the impact of this variable on the received RSRP. Moreover, the size of the markers is proportional to the duration of the file transfer, values that are extracted from the last column of Table 6 . 


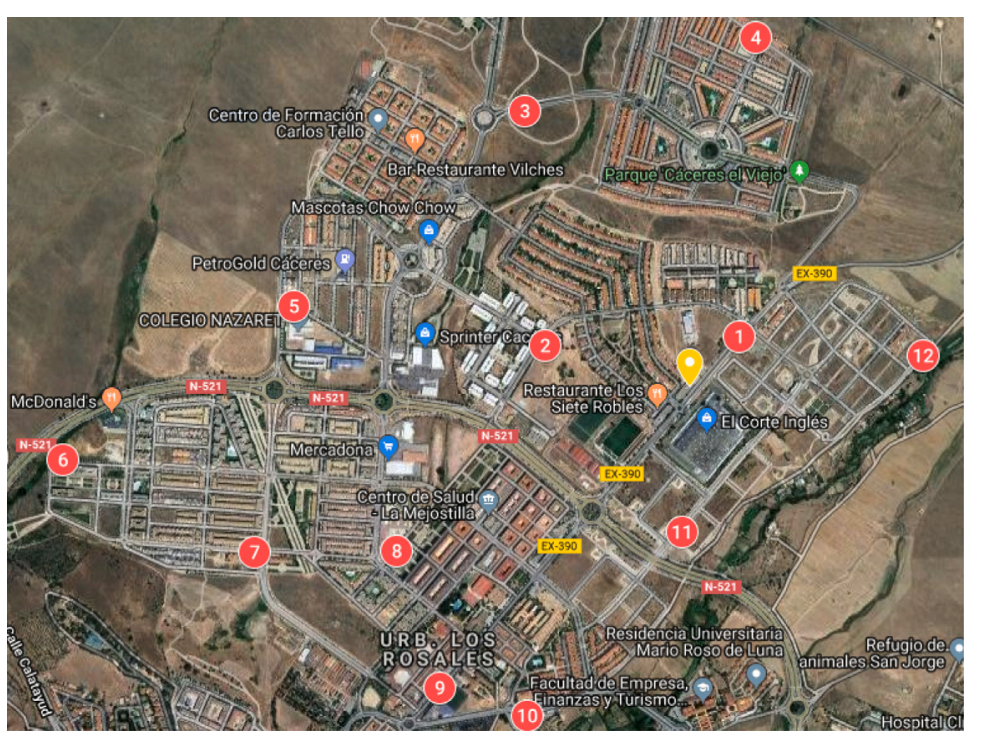

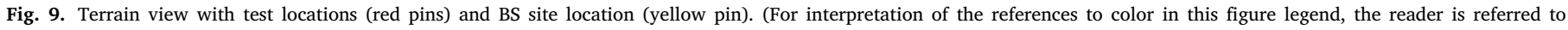
the web version of this article.)

Table 6

\begin{tabular}{|c|c|c|c|c|c|c|}
\hline $\begin{array}{l}\text { Meas. } \\
\text { ID }\end{array}$ & $\begin{array}{l}\text { RSRP } \\
{[\mathrm{dBm}]}\end{array}$ & $\begin{array}{l}\text { Distance to BS } \\
{[\mathrm{m}]}\end{array}$ & $\begin{array}{l}\text { Sight } \\
\text { Condition }\end{array}$ & $\begin{array}{l}\text { Avg. BS } \\
\text { EMF }[\mathrm{V} / \mathrm{m}]\end{array}$ & $\begin{array}{l}\text { Avg. UE } \\
\text { EMF }[\mathrm{V} / \mathrm{m}]\end{array}$ & $\begin{array}{l}\text { Transfer } \\
\text { time [s] }\end{array}$ \\
\hline 1 & -78.3 & 201.2 & LOS & 0.57 & 3.93 & 12 \\
\hline 2 & -75.9 & 435.7 & LOS & 0.50 & 1.28 & 10 \\
\hline 3 & -89.2 & 929.1 & LOS & 0.33 & 5.92 & 10 \\
\hline 4 & -116.1 & 1034.3 & LOS & 0.71 & 3.59 & 11 \\
\hline 5 & -89.5 & 1172.4 & SLOS & 0.28 & 7.42 & 15 \\
\hline 6 & -99.8 & 1847.5 & NLOS & 0.31 & 6.02 & 12 \\
\hline 7 & -100.3 & 1361.9 & NLOS & 0.29 & 7.66 & 14 \\
\hline 8 & -102.5 & 978.1 & NLOS & 0.20 & 7.89 & 18 \\
\hline 9 & -104.2 & 1143.2 & SLOS & 0.33 & 3.42 & 10 \\
\hline 10 & -76.3 & 1075.4 & NLOS & 0.70 & 2.03 & 13 \\
\hline 11 & -82.7 & 426.8 & LOS & 0.39 & 1.44 & 9 \\
\hline 12 & -83.7 & 684.3 & SLOS & 0.36 & 3.44 & 11 \\
\hline
\end{tabular}

At first, it can be noticed that in general, better RSRP values are obtained in CC than in TMC, especially when the UE is close to the BS and there are no obstacles between it and the BS (left upper side of Figs. 10(a) and 10(b)). Indeed, the lowest RSRP values are experienced in the range $[1,000-2,000][\mathrm{m}]$, especially for NLOS condition (see red square markers in Fig. 10(b)). Another important aspect to remark is that, as soon as we move far away the BS, the time required to successfully transmit the file is highly increased (note the size of the red square markers in Fig. 10(b)). In this case, CC outperforms TMC, by reducing the time more than 3 points on average. Finally, the SLOS condition does not seem to affect too much on the RSRP value nor on the time for the file sending, resulting in medium values for both metrics.

The next analysis we propose aims at comparing the EMF generated by the UE with the one produced by the serving BS, as a function of the distance, for both scenarios. In Fig. 11, to subfigures are represented, one per scenario (CC in Fig. 11(a) and TMC in Fig. 11(b)), with the distance from the serving $\mathrm{BS}$ as $\mathrm{X}$ axis. The left $\mathrm{Y}$ axis shows the value of the EMF generated by the BS when no other sources are in proximity, while the right $\mathrm{Y}$ axis reports the EMF generated by the UE during the transfer file. The sight condition is represented by different marker types (LOS - circle, Semi-LOS — diamond, and NLOS - square) and, as in Fig. 10, the size of the markers is proportional to the duration of the file transfer.
The first aspect to highlight is that the EMF values generated by the BS are, in general, much lower than the ones derived from the UE, with a maximum of $.8[\mathrm{~V} / \mathrm{m}]$ in the worst-case scenario. In case of LOS between the EMF meter and the serving BS, the EMF generated by the UE (red markers) is much lower in TMC than in CC, for all values of distance. Moreover, a decreasing logarithmic function can be extracted from the BS EMF in TMC, i.e., the average BS EMF decreases with the distance towards the serving BS. In this case, EMF values are similar than the ones reported in the CC scenario. If we now move our attention to SLOS and NLOS conditions, two main aspects emerge: (i) the average UE EMF is much lower in TMC, becoming negligible in most of the cases; and (ii) although the average BS EMF is also lower in TMC, there is not a big difference with CC as in the case of the UE EMF.

Finally, a correlation analysis between the RSRP value and the distance to the serving BS is shown in Table 7, along with their $p$ values. From the table, we can extract that there is a strong correlation (approaching to -1 ) between the RSRP and the distance in the case of LOS, i.e., when there are not obstacles in the path. As it can be seen, the correlation is negative, which means that RSRP values decrease with the distance. Moreover, although it is also negative for the NLOS condition, the correlation is not strong enough as in the case of LOS. Interestingly, SLOS presents positive correlations between variables, reaching the maximum in the $\mathrm{CC}$ scenario. 


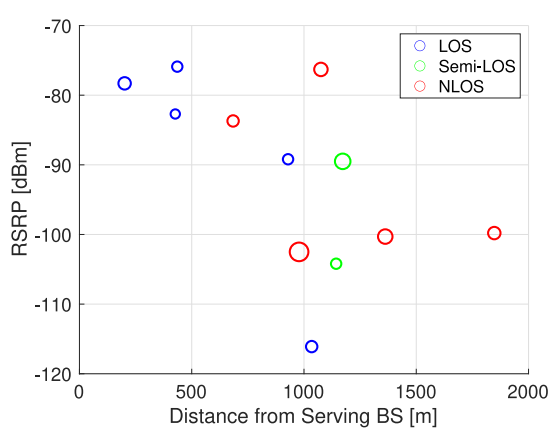

(a) $\mathrm{CC}$

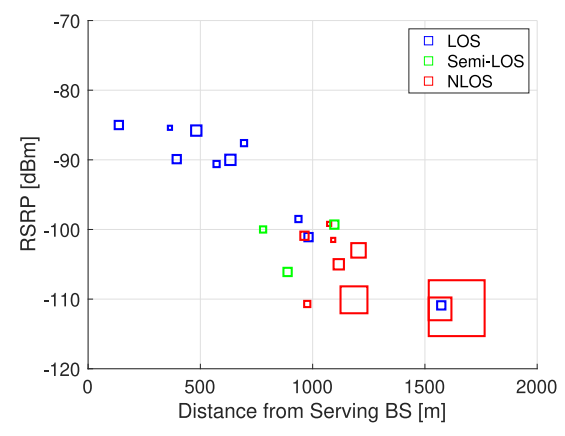

(b) TMC

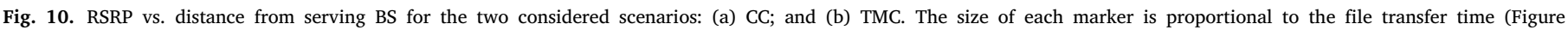
best-viewed in colors).

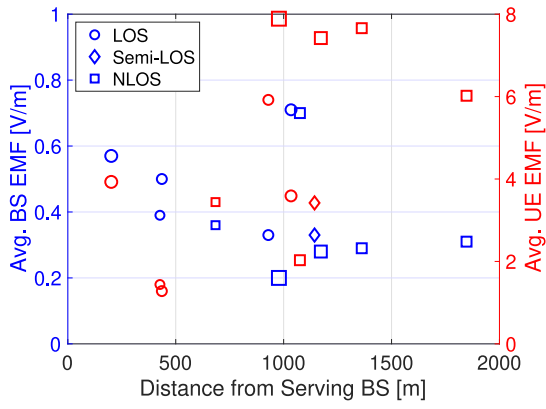

(a) $\mathrm{CC}$

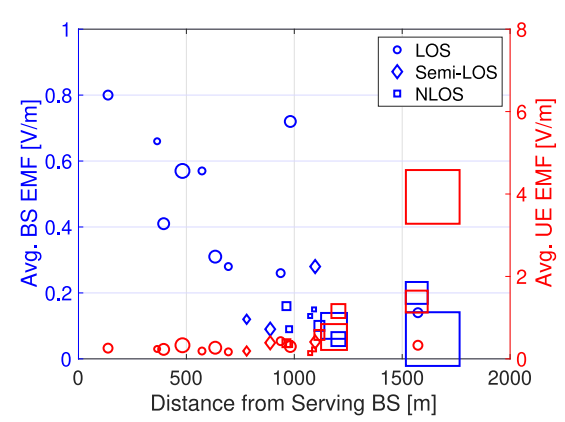

(b) TMC

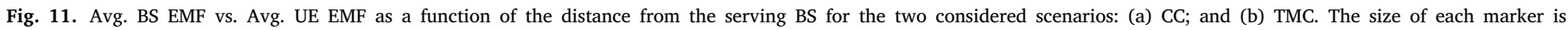
proportional to the file transfer time (Figure best-viewed in colors).

Table 7

Correlation coefficient and $p$-value for the RSRP versus distance to BS in the different scenarios.

\begin{tabular}{lll}
\hline Sight condition & CC & TMC \\
\hline LOS & $-0.8309\left(<10^{-9}\right)$ & $-0.9471\left(<10^{-9}\right)$ \\
SLOS & $1.0\left(<10^{-9}\right)$ & $0.2689\left(<10^{-9}\right)$ \\
NLOS & $-0.4982\left(<10^{-9}\right)$ & $-0.6031\left(<10^{-9}\right)$ \\
\hline
\end{tabular}

As a summary, taking into account that the RSRP is a metric that captures the constant power radiated by the BS, which does not depend on the load of the cell and it is not influenced by other constraints (e.g., the minimization of the energy due to data transmission) [27], our results prove that the stringent regulations applied in the TMC scenario generate coverage holes and bad performance indicators in different locations compared to CC, thus demonstrating that the stringent regulations have a great impact on the planning.

\section{Multivariate analysis}

In order to analyze the relationship among the considered BSrelated metrics (RSRP, sight condition, distance from serving BS), QoSrelated metrics (time to transfer a given file), and exposure levels (EMF from the BS and from the UE), as well as their impact, a set of multivariate analyses have been performed. Multivariate analysis is the statistical study of data where multiple measurements are made on each experimental unit and where the relationships among multivariate measurements and their structure are important [29].
In particular, we consider three independent variables: (i) the RSRP obtained by the UE, (ii) the distance between the UE and the BS, and (iii) the sight condition (LOS, SLOS, NLOS). Since the main goal of performing a multivariate analysis is to know the impact of each independent variable over a dependent variable, three different dependent variables have been evaluated: (i) the average EMF from the BS (EMFBS variable), (ii) the average UE EMF (EMF-UE variable), and (iii) the time to transfer the file (Time variable). As a summary, all the variables used in the multivariate analysis are defined and categorized in Table 8.

Regarding the type of variables, all of them are continuous except the ones used to categorize the sight condition (LOS, SLOS and NLOS), which are considered as categorical. In this case, LOS is taken as the reference variable in order to compare the other two with it, i.e., the case of a direct line of sight between the UE and the BS.

Applying multivariate or multiple regression analysis to a set of data results in what are known as regression coefficients, one for each independent variable. These coefficients give the estimated change in the dependent variable associated with a unit change in the corresponding independent variable, conditional on the other independent variables remaining constant. As a result, three different multivariate analyses have been performed for each scenario (CC and TMC), one for each dependent variable (EMF-BS, EMF-UE and Time).

Table 9 shows the outcomes after performing the Ordinary Least Squares (OLS) regression for EMF-BS dependent variable, with a value of the multiple correlation coefficient, $R^{2}=.924$. This value for the multiple correlation coefficient indicates that there is a strong correlation between the observed data and those predicted by the regression model. The output shown in Table 9 provides estimates of 
Table 8

Criteria and variables used in the multivariate analysis.

\begin{tabular}{lll}
\hline Variable name & Description & Type \\
\hline RSRP & RSRP value & Independent/Continuous \\
\hline Distance & Distance between UE and BS & Independent/Continuous \\
\hline LOS & $\begin{array}{l}\text { Situation where the UE is } \\
\text { in LOS with the BS }\end{array}$ & Independent/Categorical \\
\hline SLOS & $\begin{array}{l}\text { The LOS between the UE and the BS } \\
\text { is partially covered by foliage }\end{array}$ & Independent/Categorical \\
\hline EMFS & $\begin{array}{l}\text { The LOS between the UE and the BS } \\
\text { is completely obstructed by buildings }\end{array}$ & Independent/Categorical \\
\hline EMF-UE & Average BS EMF value & Dependent/Continuous \\
\hline Time & Average UE EMF value & Dependent/Continuous \\
\hline
\end{tabular}

Table 9

OLS regression results with EMF-BS as dependent variable.

\begin{tabular}{|c|c|c|c|c|c|c|c|c|c|c|}
\hline \multirow[t]{3}{*}{ Model } & \multicolumn{4}{|c|}{ Unstandardized coefficients } & \multirow{2}{*}{\multicolumn{2}{|c|}{$\begin{array}{l}\text { Std. coefficients } \\
\text { Beta }\end{array}$}} & \multirow{2}{*}{\multicolumn{2}{|c|}{$\mathrm{t}$}} & \multirow{2}{*}{\multicolumn{2}{|c|}{ Sig. }} \\
\hline & \multicolumn{2}{|l|}{$\mathrm{B}$} & \multicolumn{2}{|c|}{ Std. Error } & & & & & & \\
\hline & $\mathrm{CC}$ & TMC & CC & TMC & $\mathrm{CC}$ & TMC & $\mathrm{CC}$ & TMC & $\mathrm{CC}$ & TMC \\
\hline 1 (Cons.) & 0.618 & 0.842 & 0.443 & 0.725 & & & 1.396 & 1.161 & 0.000 & 0.000 \\
\hline RSRP & 0.001 & 0.003 & 0.006 & 0.009 & 0.110 & 0.099 & 0.252 & 0.282 & 0.000 & 0.000 \\
\hline Distance & $1.39 \cdot 10^{-5}$ & 0.000 & 0.000 & 0.000 & 0.037 & -0.347 & 0.067 & -1.095 & 0.000 & 0.001 \\
\hline SLOS & -0.191 & -0.236 & 0.180 & 0.109 & -0.443 & -0.356 & -1.062 & -2.161 & 0.013 & 0.000 \\
\hline NLOS & -0.130 & -0.215 & 0.159 & 0.094 & -0.400 & -0.466 & -0.820 & -2.280 & 0.002 & 0.000 \\
\hline
\end{tabular}

the regression coefficients, standard errors of the estimates, $t$-tests that a coefficient takes the value zero, and confidence intervals. First, it can be seen that all the variables considered in the analysis are statistically significant $(<.05$ in all cases). The estimated regression coefficients are given under the heading "Unstandardized Coefficients | B"; these give, for each of the independent variables, the predicted change in the dependent variable when the independent variable is increased by one unit conditional on all the other variables in the model remaining constant. For instance, results shown in Table 9 estimate that the EMF generated by the BS in CC is increased by $0.001[\mathrm{~V} / \mathrm{m}]$ for every additional increase of one unit in the value of the RSRP $(1[\mathrm{dBm}]$ in this case). Similarly, the estimated effect in case of TMC is an increase of $0.003[\mathrm{~V} / \mathrm{m}]$ per $[\mathrm{dBm}]$ in the value of the RSRP. Therefore, although there exists a directly proportional relationship between the RSRP value and the EMF generated by the BS, it can be seen that the impact is minimal. If we move our attention to the distance between the UE and the serving BS, a slight impact on the EMF is experienced when the distance increases $\left(1.39 \cdot 10^{-5}[\mathrm{~V} / \mathrm{m}]\right.$ per meter for CC and no impact at all in the case of TMC).

EMF is also analyzed when different sight conditions are taken into account. These categorical variables (SLOS and NLOS) are compared with the situation in which where is a line of sight between the UE and the BS (LOS), i.e., no obstacles are disturbing the communication. In particular, both SLOS and NLOS situations have an associated decrease in the experienced EMF (about $-0.1[\mathrm{~V} / \mathrm{m}]$ for CC and below -0.2 $[\mathrm{V} / \mathrm{m}]$ for TMC) compared with the LOS condition. As a summary, from the results obtained after performing the OLS taking EMF-BS as dependent variable, we can extract that the considered variables have not a strong impact on the EMF that is generated solely by the serving BS.

The explanation for the remaining columns in the table is provided next. The column "Unstandardized Coefficients | Std. Error" shows the standard error for each of the regression coefficients described above. The third column shows the standardized regression coefficients under the heading "Std. Coefficients | Beta". These coefficients are standardized so that they measure the change in the dependent variable in units of its standard deviation when the independent variable increases by one standard deviation. Finally, the last two columns represent the $t$-tests and the significance value.
Table 10 shows the multivariate analysis performed with EMF-UE as dependent variable. In this case, the impact of SLOS and NLOS conditions compared with the LOS reference variable is remarkable in the CC scenario, with more than $1[\mathrm{~V} / \mathrm{m}]$ in the EMF generated by the UE. On the contrary, there is no difference among the three sight conditions on the EMF in the TMC scenario. Indeed, the impact of the RSRP and the distance to the serving BS on the EMF generated by the UE is also minimal in both scenarios.

Finally, we analyze by inspecting Table 11 the impact on the time required to upload the file (Time variable). As in the previous analysis, the sight condition is the variable that impacts most, for which the time to transfer the file increases up to about 4 times compared to the case in which there is LOS with the BS. As in the previous OLS, the value of the RSRP and the distance to the serving BS slightly impact the time needed for the file transfer.

As a summary, the set of OLS regressions performed reinforces the analyses described in previous sections. In particular, the main conclusions we extract are: (i) there is not a strong impact of the variables used in the analyses on the EMF generated solely by the serving BS; (ii) the sight condition clearly impacts the EMF generated by the UE, especially in ICNIRP-based scenarios; (iii) the sight condition is fundamental when the time required to send the file is evaluated, with similar impact in both scenarios.

\section{Conclusions and future work}

In this paper, a comparison of the cellular network planning that is deployed in two countries with different EMF restrictions (Spain - ICNIRP, Italy, stricter-than-ICNIRP) is performed. In particular, two analyses have been carried out from a technical point of view to compare the network planning of already-deployed 4G cellular networks in two similar districts in terms of terrain dimensions and population density: (i) a quantitative analysis of QoS metrics perceived by the user; and (ii) a qualitative analysis on the EMF generated by the UE and the perceived QoS.

Regarding the first analysis, we remark that there is a strong difference in the RSRP values obtained in similar districts located in different countries with different regulations, with the corresponding impact on 
Table 10

OLS regression results with EMF-UE as dependent variable.

\begin{tabular}{|c|c|c|c|c|c|c|c|c|c|c|}
\hline \multirow[t]{3}{*}{ Model } & \multicolumn{4}{|c|}{ Unstandardized coefficients } & \multirow{2}{*}{\multicolumn{2}{|c|}{$\begin{array}{l}\text { Std. coefficients } \\
\text { Beta }\end{array}$}} & \multirow{2}{*}{\multicolumn{2}{|c|}{$\mathrm{t}$}} & \multirow{2}{*}{\multicolumn{2}{|c|}{ Sig. }} \\
\hline & \multicolumn{2}{|l|}{ B } & \multicolumn{2}{|c|}{ Std. Error } & & & & & & \\
\hline & $\mathrm{CC}$ & TMC & $\mathrm{CC}$ & TMC & $\mathrm{CC}$ & TMC & $\mathrm{CC}$ & TMC & $\mathrm{CC}$ & TMC \\
\hline 1 (Cons.) & -2.378 & 2.081 & 5.689 & 3.592 & & & -0.418 & 0.579 & 0.000 & 0.000 \\
\hline RSRP & -0.056 & 0.031 & 0.073 & 0.044 & -0.299 & 0.351 & -0.763 & 0.707 & 0.000 & 0.000 \\
\hline Distance & 0.001 & 0.002 & 0.003 & 0.001 & 0.213 & 0.782 & 0.423 & 1.739 & 0.000 & 0.000 \\
\hline SLOS & 1.096 & -0.045 & 2.307 & 0.540 & 0.177 & -0.019 & 0.475 & -0.083 & 0.000 & 0.000 \\
\hline NLOS & 1.289 & 0.272 & 2.041 & 0.467 & 0.276 & 0.169 & 0.632 & 0.582 & 0.000 & 0.000 \\
\hline
\end{tabular}

Table 11

OLS regression results with Time as dependent variable.

\begin{tabular}{|c|c|c|c|c|c|c|c|c|c|c|}
\hline \multirow[t]{3}{*}{ Model } & \multicolumn{4}{|c|}{ Unstandardized coefficients } & \multirow{2}{*}{\multicolumn{2}{|c|}{$\begin{array}{l}\text { Std. coefficients } \\
\text { Beta }\end{array}$}} & \multirow{2}{*}{\multicolumn{2}{|c|}{$\mathrm{t}$}} & \multirow{2}{*}{\multicolumn{2}{|c|}{ Sig. }} \\
\hline & \multicolumn{2}{|l|}{$\mathrm{B}$} & \multicolumn{2}{|c|}{ Std. Error } & & & & & & \\
\hline & $\overline{\mathrm{CC}}$ & TMC & $\overline{\mathrm{CC}}$ & TMC & $\overline{\mathrm{CC}}$ & TMC & $\overline{\mathrm{CC}}$ & TMC & $\overline{\mathrm{CC}}$ & TMC \\
\hline 1 (Cons.) & 6.469 & 11.826 & 5.982 & 64.204 & & & 1.082 & 0.184 & 0.000 & 0.000 \\
\hline RSRP & -0.057 & 0.169 & 0.077 & 0.790 & -0.285 & 0.110 & -0.741 & 0.215 & 0.000 & 0.000 \\
\hline Distance & -0.002 & 0.020 & 0.003 & 0.016 & -0.319 & 0.556 & -0.646 & 1.191 & 0.000 & 0.000 \\
\hline SLOS & 2.623 & -3.603 & 2.426 & 9.655 & 0.397 & -0.091 & 1.081 & -0.373 & 0.000 & 0.000 \\
\hline NLOS & 4.026 & 3.493 & 2.146 & 8.350 & 0.805 & 0.126 & 1.876 & 0.418 & 0.000 & 0.000 \\
\hline
\end{tabular}

the QoS perceived by the users. In particular, the ICNIRP-based scenario presents, in general, much better values of RSRP for all the operators compared to the case of the stricter-than-ICNIRP one. Moreover, a reduction in the number of BSs (and hence in the number of cells) per portion of territory is also experienced in the ICNIRP scenario.

Concerning the evaluation of the impact of the generated EMF in the two scenarios, it is highlighted that (i) EMF values generated by BSs are, in general, much lower than the ones generated by the UE; (ii) EMF values in the stricter-than-ICNIRP scenario are overall lower compared to the case of the ICNIRP scenario; (ii) the sight condition between the UE and the BS clearly impacts the EMF generated by the UE, especially in the ICNIRP-based district; and (iii) the sight condition is also fundamental when evaluating the impact on the uplink performance.

As future works, in order to strengthen our conclusions, we plan to evaluate the impact of the network planning in terms of QoS and EMF in indoor environments, as well as the type of traffic required by the applications (elastic, non-elastic) on the EMF generated by the UE.

\section{CRediT authorship contribution statement}

Jaime Galán-Jiménez: Conceptualization, Methodology, Software, Validation, Formal analysis, Investigation, Resources, Data curation, Writing - original draft, Writing - review \& editing, Visualization, Supervision, Project administration, Funding acquisition. Luca Chiaraviglio: Conceptualization, Methodology, Software, Validation, Formal analysis, Investigation, Resources, Data curation, Writing - original draft, Writing - review \& editing, Visualization, Supervision, Project administration, Funding acquisition.

\section{Declaration of competing interest}

The authors declare that they have no known competing financial interests or personal relationships that could have appeared to influence the work reported in this paper.

\section{Acknowledgments}

This work has been partially funded by the project RTI2018-094591-B-I00 (MCI/AEI/FEDER,UE, Spain), the 4IE+ Project (0499-4IEPLUS-4-E) funded by the Interreg V-A España-Portugal, Spain (POCTEP) 2014-2020 program, and by the Department of Economy, Science and Digital Agenda of the Government of Extremadura, Spain (GR18112, IB18030). The authors would also thank all the people involved in the measurement campaign over the CC scenario.

\section{References}

[1] A. Ahlbom, U. Bergqvist, J. Bernhardt, J. Cesarini, M. Grandolfo, M. Hietanen, A. Mckinlay, M. Repacholi, D. Sliney, J.A. Stolwijk, M.L. Swicord, L.D. Szabo, M. Taki, T.S. Tenforde, H.P. Jammet, R. Matthes, Guidelines for limiting exposure to time-varying electric, magnetic, and electromagnetic fields (up to $300 \mathrm{GHz}$ ), Health Phys. 74 (4) (1998) 494-521.

[2] Exposure limits for radio-frequency fields (public) - data by country, 2021, Available at http://apps.who.int/gho/data/node.main. EMFLIMITSPUBLICRADIOFREQUENCY?lang=en. (Last Accessed 7 January 2021).

[3] Regolamento per la localizzazione, l'installazione e la modifica degli impianti di telefonia mobile, ai sensi dell'art. 8, comma 6, della legge n. 36 del 22 febbraio 2001 e per la redazione del Piano, ex art. 105, comma 4 delle NTA del PRG vigente, nonché per l'adozione di un sistema di monitoraggio delle sorgenti di campo elettrico, magnetico ed elettromagnetico, 2021 (in Italian), Available at https://www.comune.roma.it/web-resources/cms/documents/DACDelib_N_26_ 14.05.2015.pdf. (Last Accessed 7 January 2021).

[4] TESTO COORDINATO DEL DECRETO-LEGGE 18 ottobre 2012, n. 179 Ulteriori misure urgenti per la crescita del Paese, 2021 (in Italian), Available at www. gazzettaufficiale.it/eli/id/2012/12/18/12A13277/sg. (Last Accessed 7 January 2021).

[5] Base station planning permission in Europe, 2021, Available at https://www.gsma.com/publicpolicy/wp-content/uploads/2013/05/GSMA_ BaseStation_Planning_EuropeWEB.pdf. (Last Accessed 7 January 2021).

[6] S. Sagar, S. Dongus, A. Schoeni, K. Roser, M. Eeftens, B. Struchen, M. Foerster, N. Meier, S. Adem, M. Röösli, Radiofrequency electromagnetic field exposure in everyday microenvironments in Europe: A systematic literature review, J. Expo. Sci. Environ. Epidemiology 28 (2017) http://dx.doi.org/10.1038/jes.2017.13.

[7] Impact of EMF limits on 5G network roll-out, 2021, Available at https://www.itu.int/en/ITU-T/Workshops-and-Seminars/20171205/Documents/ S3_Christer_Tornevik.pdf. (Last Accessed 7 January 2021).

[8] Implications of RF-EMF exposure limits for 5G: lessons from $3 G$ and $4 G$ deployments, 2021, Available at https://www.itu.int/en/ITU-T/Workshops-andSeminars/20171205/Documents/S3_Jack_Rowley.pdf. (Last Accessed 7 January 2021).

[9] L. Chiaraviglio, A.S. Cacciapuoti, G.D. Martino, M. Fiore, M. Montesano, D. Trucchi, N.B. Melazzi, Planning $5 \mathrm{G}$ networks under EMF constraints: State of the art and vision, IEEE Access 6 (2018) 51021-51037.

[10] IARC classifies radiofrequency electromagnetic fields as possibly carcinogenic to humans, 2021, Available at http://www.iarc.fr/en/media-centre/pr/2011/pdfs/ pr208_E.pdf. (Last Accessed 7 January 2021).

[11] National Toxicology Program releases final reports on rat and mouse studies of radio frequency radiation like that used in $2 \mathrm{G}$ and $3 \mathrm{G}$ cell phone technologies, 2021, Available at https://www.niehs.nih.gov/news/newsroom/releases/2018/ november1/index.cfm. (Last Accessed 7 January 2021).

[12] M.E. Wyde, T.L. Horn, M.H. Capstick, J.M. Ladbury, G. Koepke, P.F. Wilson, G.E. Kissling, M.D. Stout, N. Kuster, R.L. Melnick, et al., Effect of cell phone radiofrequency radiation on body temperature in rodents: Pilot studies of the National Toxicology Program's reverberation chamber exposure system, Bioelectromagnetics 39 (3) (2018) 190-199. 
[13] L. Falcioni, L. Bua, E. Tibaldi, M. Lauriola, L. De Angelis, F. Gnudi, D. Mandrioli, M. Manservigi, F. Manservisi, I. Manzoli, I. Menghetti, S. Montella, D. Sgargi, V. Strollo, V. A., F. Belpoggi, Report of final results regarding brain and heart tumors in Sprague-Dawley rats exposed from prenatal life until natural death to mobile phone radiofrequency field representative of a $1.8 \mathrm{GHz}$ GSM base station environmental emission, Environ. Res. 165 (2018) 496-503.

[14] L. Chiaraviglio, M. Fiore, E. Rossi, 5G technology: Which risks from the health perspective?, in: The 5G Italy Book 2019: A Multiperspective View of 5G, CNIT, 2019.

[15] ICNIRP guidelines for limiting exposure to electromagnetic fields (100 kHz to $300 \mathrm{GHz}), 2021$, Available at https://www.icnirp.org/cms/upload/publications/ ICNIRPrfgdl2020.pdf. (Last Accessed 7 January 2021).

[16] GSMA. EMF policy. Public policy (network limits tab), 2021, Available at https:// www.gsma.com/publicpolicy/consumer-affairs/emf-and-health/emf-policy. (Last Accessed 7 January 2021).

[17] ITU-T K.Sup14 : The impact of RF-EMF exposure limits stricter than the ICNIRP or IEEE guidelines on 4G and 5G mobile network deployment, 2021, Available at https://www.itu.int/rec/T-REC-K.Sup14-201805-I. (Last Accessed 7 January 2021).

[18] DELIBERAZIONE DELLA GIUNTA REGIONALE 5 settembre 2005, n.16757, 2021 (in Italian), Available at http://www.regione.piemonte.it/ambiente/ elettromagnetismo/dwd/normativa/regionale/dgr_05_09_05.pdf. (Last Accessed 7 January 2021)

[19] Cellmapper, 2021, Available at https://www.cellmapper.net/. (Last Accessed 7 January 2021).

[20] Informe de Población del Ayuntamiento de Cáceres, 2019, 2021 (in Spanish), Available at https://sig.caceres.es/descargassig/estadisticas_de_poblacion/ Informe_Poblacion_a_1_de_Enero_2019.pdf, (Last Accessed 7 January 2021).

[21] Elenco Suddivisioni Territoriali Toponomastiche - Roma Capitale, 2021 (in Italian), Available at https://www.comune.roma.it/web-resources/cms/documents/ ElencoSuddivisioniToponomastiche_rg_A.pdf, (Last Accessed 7 January 2021).

[22] Popolazione - Roma Capitale, 2021 (in Italian), Available at https: //www.comune.roma.it/web-resources/cms/documents/Tav_9_SudTop_T_18.xlsx, (Last Accessed 7 January 2021).

[23] L. Chiaraviglio, J. Galán-Jiménez, M. Fiore, N. Blefari-Melazzi, Not in my neighborhood: A user equipment perspective of cellular planning under restrictive EMF limits, IEEE Access 7 (2019) 6161-6185.

[24] MapMarker, 2021, Available at https://www.mapmarker.app/. (Last Accessed 7 January 2021).

[25] CellMapper eNB calculator, 2021, Available at https://www.cellmapper.net/ enbid. (Last Accessed 7 January 2021).

[26] Pandas: Python Data Analysis Library, 2021, Available at https://pandas.pydata. org/. (Last Accessed 7 January 2021).

[27] C. Ide, R. Falkenberg, D. Kaulbars, C. Wietfeld, Empirical analysis of the impact of LTE downlink channel indicators on the uplink connectivity, in: Vehicular Technology Conference (VTC Spring), 2016 IEEE 83rd, IEEE, 2016, pp. 1-5.

[28] PMM 8053B EMF Meter, 2021, Available at http://www.gruppompb.uk.com/ public/upload/8053BEN-40918-3.16.pdf. (Last Accessed 7 January 2021).

[29] I. Olkin, A. Sampson, Multivariate analysis: Overview, in: N.J. Smelser, P.B. Baltes (Eds.), International Encyclopedia of the Social \& Behavioral Sciences, Pergamon, Oxford, 2001, pp. 10240-10247, http://dx.doi.org/10.1016/B0-08043076-7/00472-1.

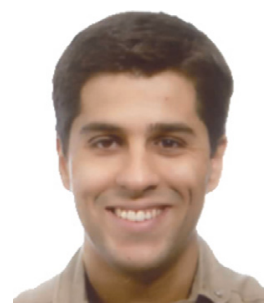

Jaime Galán-Jiménez received his Ph.D in Computer Science and Communications from the University of $\mathrm{Ex}$ tremadura (Spain) in 2014. He is currently working at the Department of Computer Systems and Telematics Engineering, University of Extremadura, as an assistant professor. In 2018, he was awarded with the Teaching Excellence Award from the University of Extremadura. During the past years, he has spent several research and teaching periods at University of Rome Tor Vergata, and at University of Rome La Sapienza, Italy. His main research interests are Software-Defined Networks, Traffic Matrix estimation, 5G networks planning and design, 5G provisioning in rural and low-income areas and Mobile Ad-Hoc Networks.

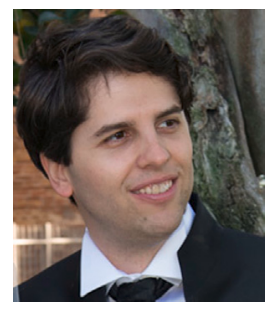

Luca Chiaraviglio (M'09-SM'16) received the Ph.D. degree in telecommunication and electronics engineering from the Politecnico di Torino, Italy. During the past years, he has spent research periods with Boston University, Boston, MA, USA; INRIA Sophia Antipolis, France; Auckland University of Technology, New Zealand; and ETECSA S.A., Cuba. He is currently a Tenure Track Assistant Professor with the Department of Electronic Engineering, University of Rome Tor Vergata, Italy. He has coauthored over 130 papers published in international journals, books, and conferences, and has collaborated with over 150 coauthors, which are affiliated with more than 40 national and international institutions. His current research interests include $5 \mathrm{G}$ networks, cloud computing, optimization applied to telecommunication networks, new architectures to reduce the digital divide in rural and low-income areas, and electromagnetic fields. $\mathrm{He}$ is currently the coordinator of the national project BRIGHT: Bringing 5G Connectivity in Rural and Low-Income Areas. During the last years, he has been involved in different European projects, such as the H2020 5G-EVE, H2020 Superfluidity, FP7 Trend, FP7 EcoNet, and FP7 Bone. $\mathrm{He}$ is a founding member of the IEEE Communications Society Technical Subcommittee on Green Communications and Computing. According to Google Scholar, his H-Index is 30. He participates in the TPC of top-leading conferences, including the IEEE INFOCOM, the IEEE GLOBECOM, the IEEE ICC, the IEEE VTC, and the IEEE GlobalSIP. He is a member of the organizing committee of several conferences, such as ECOC, LANMAN, and 5G-Italy. He is in the Editorial Board of the IEEE Communications Magazine, IEEE ACCESS, and the IEEE TRANSACTIONS ON GREEN COMMUNICATIONS AND NETWORKING. He has received the Best Paper Award in different conferences, including the IEEE VTC and ICIN. Some of his papers are listed as Best Readings on Green Communications by IEEE. Moreover, he has been recognized as an author in the top 1 most highly cited papers in the ICT field worldwide. His papers "Optimal Energy Savings in Cellular Access Networks" and "Reducing Power Consumption in Backbone Networks" are the most cited papers from all the IEEE ICC conferences and the IEEE ICC workshops, from 2009 to 2018 (Source: Scopus). 